\title{
APPLICATION OF THE METHODS OF HYBRID ARTIFICIAL INTELLIGENCE TO DETERMINE THE LOCATION OF OBJECTS BY THEIR PHOTOGRAPHY
}

\author{
Dmitry V. Berezkin ${ }^{1,4}$, Andrew V. Proletarsky², Valentin Yu. Sidorov ${ }^{3,4}$ and Ilya E. Sergeev ${ }^{3,4}$ \\ ${ }^{I}$ Ph. D., associate Professor \\ ${ }^{2}$ Doctor of Technical Sciences, Professor, Dean of the Faculty of Informatics and Control Systems, Bauman Moscow \\ State Technical University 5c1, $2^{\text {nd }}$ Baumanskaya st., 105005, Moscow, Russian Federation \\ ${ }^{3}$ Master's degree student \\ ${ }^{4}$ Department of Computer systems and networks, Bauman Moscow State Technical University \\ 5c1, $2^{\text {nd }}$ Baumanskaya st., 105005, Moscow, Russian Federation
}

\begin{abstract}
Successful assessment of the geolocation of photography can be used in many interesting projects, although this is an extremely difficult task. The article is devoted to the development of an approach to the creation of a hybrid intellectual system for determining the location of objects from their photographs. The analysis of existing approaches to the assessment of geolocation of photographs was carried out; a generalized structure of the hybrid intelligent system for determining the location of objects from their photographs was proposed. To assess the location, it is proposed to analyze both the data on the GPS coordinates of the original photo and those similar to it, and the classification of objects in the photo: city, nature. Geolocation is considered as a classification problem when the earth's surface is divided into disjoint adjacent cells.
\end{abstract}

\section{KEYWORDS}

Geolocation Estimation, Scene Classification, Hybrid Artificial Intelligence, Computer Vision, Digital Image Processing Neural Network, Deep Learning

\section{INTRODUCTION}

In recent years, methods of hybrid artificial intelligence have been intensively developed, making it possible to take advantage of traditional means and methods of artificial intelligence, and, at the same time, to overcome their inherent flaws, which are capable of solving problems that have so far been unsatisfactorily solved by separate methods of artificial intelligence. Hybrid intelligent systems (HIS) allow the most efficient processing of formalized and non-formalized knowledge through the integration of both traditional data processing methods and artificial intelligence - expert and fuzzy methods, artificial neural networks, genetic algorithms, etc. (Dilip Kumar Choubey and Sanchita Paul, 2016; Adane Nega and Alemu Kumlachew, 2017; Bobkov, A.V. and Yang, Xu., 2019).

Establishment of such a system is the object of this study.

In many situations, locating objects from their photographs is required. If the photo is taken with a modern digital camera with a built-in GPS sensor that captures the coordinates of the object, then the problem is solved quite simply. The coordinates of the object are placed in hidden photo metadata, which are clearly invisible, but can be obtained using specialized software.

However, the availability of metadata about the location of an object in a photograph (geolocation) depends on several factors. Firstly, from the device with which the photograph was taken. Some cameras or mobile devices may not have a built-in GPS sensor that records the coordinates of the survey. Secondly, from the desire of users of mobile devices: they can turn off geolocation for reasons of privacy or reducing the load on the battery. Thirdly, the availability of such data depends on the resource on which the photo was published. Social networks such as Facebook, Twitter or Instagram delete the metadata of photograph while they are uploaded on servers of these resources. Check the availability of metadata is pretty simple. For 
doing this, you can upload a photo file and check its properties. For faster and more efficient analysis of photo metadata contained in the EXIF data file, you can use special on-line resources.

It should be noted that in many cases photo metadata is missing or distorted. At the same time, determining the location of an object from its photograph is necessary for solving a number of tasks, and first of all when analyzing big data from various sensors. In this case, an automatic determination of the location of an object in a photograph should be performed only on the basis of the presence of the photograph itself and the analysis of its content. A preliminary analysis showed that the task of determining the location of objects from their photographs is relevant in the context of the rapid development of the Internet of Things (IOT) technology (Zaharieva, M., Zeppelzauer, M. and Breiteneder, C., 2013; Weyand, T., Kostrikov, I. and Philbin, J., 2016).

The article will consider the approach to combining various methods of artificial intelligence, which allows to solve the complex practical problem of determining the location of objects from their photographs.

\section{BACKGROUND}

At present, intellectual systems, as a rule, are not developed separately, but are embedded as modules into traditional information systems for solving problems associated with intelligent processing of data and knowledge.

In (Zaharieva, M., Zeppelzauer, M. and Breiteneder, C., 2013) the question of determining a specific event based on an array of photographs is considered. However, it is noted that metadata, in particular, containing geolocation, can often be incomplete and unreliable. Determining the geolocation of a photo allows you to significantly simplify the process of clustering photos by event, as well as determine the geolocation of the event.

There are studies where photographs with known geolocation are used to teach a mathematical model, for example, (Suslov S. and Gavrikova K., 2017), which shows that the definition of geolocation of a photo without metadata can help expand the amount of data for subsequent analysis and improve its quality.

Google has stated that its search engine will soon be able to determine the place in which one or another photo was taken. To do this, the company's employees created a neural network called PlaNet (Weyand, T., Kostrikov, I. and Philbin, J., 2016), in the database of which more than 90 million images were loaded indicating the places in which they were taken. During the search for a place, PlaNet will compare the snapshot with images from the database at the pixel level. In case of coincidence, it will suggest where the picture was taken. The basic principles of recognition are described in article (Weyand, T., Kostrikov, I. and Philbin, J., 2016). It should be noted that while the system is far from ideal. During the first tests, the program was able to correctly identify the country only in $28.4 \%$ of cases, and the continent - in $48 \%$.

In (Nam, N. Vo, Jacobs, N. and Hays, J., 2017), the Im2GPS project is presented, in which the geolocation estimation based on a comparison of the original photo with 6 million prepared Flickr images for which GPS coordinates are given. Comparative results of the assessment of the geo-location of the projects PlaNet and Im2GPS are described in (Müller-Budack, E., Pustu-Iren, K. and Ewerth, R., 2018; Nam, N. Vo, Jacobs, N. and Hays, J., 2017).

\section{PRACTICAL IMPLEMENTATION}

Analysis of known approaches to determining the location of objects from photographs and their implementations showed the absence of ready-made, in terms of quality of recognition, ready-made solutions. In this regard, to solve practical problems, it is necessary to develop its own geolocation system, for which the following algorithm is proposed.

1. From a photo from the test suite (for which the metadata is missing or distorted) try to find a similar photo, then find its description (or analyze the surrounding context) and metadata, if any. Then extract the geolocation information by mining the collected textual information.

2. Analyze a photo from the test suite using a trained neural network (or several specialized neural networks previously trained in the ways described below). In this case, methods of computer vision (noise filtering, background removal, etc.) can be applied. As a result of the analysis, a vector of features (tags) and an 
assessment of the tag's compliance with the image are formed. A search query is formed from the tags and images are searched for in the existing image database or on the Internet. The most similar of them is determined, as well as its name and context, which is then analyzed in the manner described in paragraph 1.

3. The result of image analysis can be checked and refined using ontological reference books. As a result of the check, the quality of geolocation can be improved, some errors can be corrected and the result obtained can be substantiated.

In fig. 1 shows a generalized structure of a multi-agent system for determining the location of objects from their photographs, which implements the proposed algorithm of work.

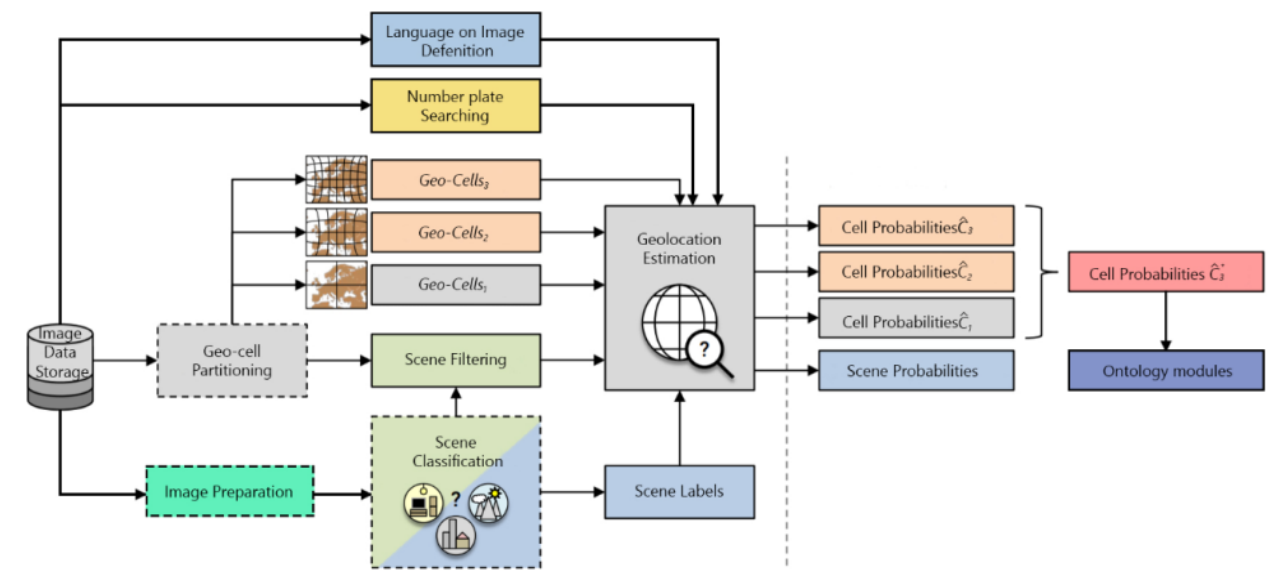

Figure 1. The generalized structure of the hybrid intelligent system for determining the location of objects from their photographs

\subsection{Image Preparation Module}

An important module for a hybrid intelligent system that uses neural networks in its work is the image preparation module. As a rule, the data for training the neural network is taken from open sources, for example, from the Flickr social network, so images may contain filters or processing added by the user, which will create errors and interfere with learning. The image preprocessing module should determine the image parameters and eliminate defects on it.

The analysis showed that the original photos used for training might contain the following defects:

- defocusing the image;

- glare, occupying more than $20 \%$ of the photos;

- too dark and too light images;

- the presence of foreign objects (dust, drops, insects, etc.);

- the presence of various filters that change the color correction of the image.

The image preparation module should take into account the following factors affecting the subsequent image processing using neural networks.

1. It is necessary to ensure the same aspect ratio of the processed images. Most neural network models accept a square input image, which means that each image needs to be checked whether it is square or not, and whether it is cropped appropriately. During this cropping, it is important to observe the required quality of the central part of the image.

2. It is necessary to analyze the average standard deviation of the input data, by which we will understand the degree of deviation of the observation data or sets from the mean value obtained by taking the average values for each pixel in all learning examples. This value can be calculated as:

$\delta=\sqrt{\frac{\sum_{j-1}^{n}\left(X_{j}-X_{c o}\right)^{2}}{n-1}}$ 
where $X_{i}$ is the pixel value, $X_{c p}$ is the average pixel value.

It gives an idea of some basic structure of images, which is used in the next stage of their processing.

3. Requires normalization of the original images entering the system.

\subsection{Text Language Definition Module}

Recognition of the language in which the text is written on the image is a very important part of the positioning system of the photo. This module allows to reduce the search to a group of countries if the recognized language is a state language for several countries or to determine a specific country if the language is unique.

The search and determination of the language in an image go through several stages. The first stage is to search for text on the image. To implement this function, you can use the computer vision methods given in article (Xinyu Zhou, Cong Yao, He Wen, Yuzhi Wang, Shuchang Zhou, Weiran He and Jiajun Liang, 2017).

After this detector has processed the image, all areas containing text information will be highlighted on the image, and the coordinates of these areas will be known. The next step is to extract the text from the image. To extract text, you can use the Tesseract engine (Thakare, S., Kamble, A., Thengne, V. and Kamble, U.R., 2018).

Preliminary results indicated that this mechanism shows high accuracy on pre-processed data, but it will not work well if there is significant noise in the image or if it is not processed and cleaned enough. Also, besides direct search and extraction of the image, it is necessary to automatically determine in which language the text is written. To do this, use the open source library - langdetect, written for the Python or Java language. This library generates language profiles based on xml annotations from Wikipedia, determines the language of the text using the Bayesian filter. Its accuracy is $99 \%$ for 53 languages.

\subsection{Geo-Cell Partitioning Module}

It is assumed that the system under development will analyze huge volumes of photos, each of which can be located anywhere in the world. Therefore, it will be advisable to divide the earth's surface into disjoint segments and analyze the belonging of photos to one or another segment, which will reduce the time spent on the system. Software solutions for segmenting the earth's surface are known, for example, GeoHash and GoogleS2. Each of these solutions implies a hierarchical division of the globe into cells, that is, there is such a thing as a cell "level". At one level, the cells are the same size.

GeoHash has a number of serious drawbacks, for example, the presence of only 12 "levels" of segmentation of the earth's surface, the spread of the distance between which reaches large values (GoogleS2 has 30). For this reason, some areas may not be adequately addressed. Also, one cannot fail to note the fact that for encoding a location using the GeoHash solution, 1 to 12 bytes of memory are required, depending on the level of segmentation, whereas for S2Geometry it is 1 byte (Deiotte, R. and La Valley, R., 2017).

Google S2 has an undeniable advantage in the form of adaptive hierarchical division of the earth's surface into cells. A more detailed description and testing of the above projects is described in (Deiotte, R. and La Valley, R., 2017).

Geometric library S2 is used to generate a set of non-overlapping geographic cells C. In more detail, the Earth's surface is projected onto an enclosing cube with six sides representing the original cells. An adaptive hierarchical unit is used based on the coordinates of GPS images, where each cell is a node of a quadrangular tree.

S2 library is in the public domain, is actively supported by Google and is used in projects (Zaharieva, M., Zeppelzauer, M. and Breiteneder, C., 2013; Thakare, S., Kamble, A., Thengne, V. and Kamble, U.R., 2018).

In the developed system, it was decided to use the dynamic separation of the earth's surface into segments using 3 "levels" of S2 library segmentation, and the "level" of a particular segment depends on the density and number of photographs located inside it. Preliminary experiments have shown that using this approach will positively affect the performance of the entire system.

In the designed HIS, it is possible to perform geolocation of only photos taken on land, so there is no need to take into account segments that are not related to the mainland surface. S2 library allows you to limit the mainland using polygons. 
Using the GeoJSON spatial data exchange format, you can quickly and conveniently convert the set of coordinates of each country into polygons, and then use them for S2Geometry. A geodata package that provides GeoJSON polygons for all countries of the world is provided by the Natural Earth service.

\subsection{Scene Classification Module}

When solving the problem of determining the location of objects from a photograph, it is necessary to correctly recognize and classify them. In addition, key and background objects in the image should be distinguished to increase recognition accuracy.

At this stage of work, the system searches for objects in photos and tags them. This is done using neural network technologies. An analysis of known approaches to solving this problem (Weyand, T., Kostrikov, I. and Philbin, J., 2016; Fedorenko, Y. S. and Gapanyuk, Y. E., 2018) showed that it is impossible to train the network so that it can successfully recognize all the signs in the image. Therefore, it was decided to use a hybrid neural network.

Thus, a universal neural network performs a general image analysis for the presence of objects belonging to different classes, for example, houses, streets - city, forest, river - nature. This will allow you to sort the images for later analysis by other neural networks.

The text-analytic neural network performs search for text data on images, their extraction and analysis. Text analysis consists in an attempt to determine the text language and, based on this, assume that the image belongs to a specific region. Also, the neural network can extract text, which allows you to form queries to search engines. For example, if you can select the name of the store in a photo, then its address in some cases can be determined based on information search results on the Internet.

The described system should use the methods of machine learning and, in particular, deep learning of its neural networks. To solve the problem of classifying an object in an image, it is proposed to use multilayer convolutional neural networks (Müller-Budack, E., Pustu-Iren, K. and Ewerth, R., 2018; Weyand, T., Kostrikov, I. and Philbin, J., 2016; Fedorenko, Y. S. and Gapanyuk, Y. E., 2018), and the training and tuning of the neural network parameters should be carried out taking into account the specifics of the arrays of the processed photos.

\subsection{Ontological Modules}

To formulate the final results of the analysis, it is proposed to use the ontological approach, which allows to clarify the results of geolocation and justify the accepted HIS conclusions.

To solve the problem of constructing ontological directories, a stage is provided for creating preliminary descriptions and their automatic conversion to the form of formal ontologies of the $\mathrm{W} 3 \mathrm{C}$ consortium, for editing which the Protégé ontology editor is used (Berezkin, D. V., 2014.). Ontologies can be stored in the form of RDF triplets in various DBMSs using the Jena system; access to them is carried out in the SPARQL language. The issues of increasing the speed of execution of SPARQL queries by their effective caching are resolved.

\section{CONCLUSION}

The article describes the existing and proposed a new approach to creating an intelligent system for determining the location of objects from their photographs, using data extracted from large databases of unstructured information. Ways of improving the accuracy of positioning are outlined by checking and refining the solutions obtained by the neural network using the classification of objects in the photograph. The introduction of the proposed technology will improve the analysis of unstructured information of social networks, solve new problems in the field of IoT and Big Data. 


\section{REFERENCES}

Adane Nega and Alemu Kumlachew, 2017. Data Mining Based Hybrid Intelligent System for Medical Application. International Journal of Intelligent Systems and Applications (IJISA), Vol.8, No.1, pp. 49-59.

Berezkin, D. V., 2014. Tekhnologiya upravleniya raznorodnymi znaniyami [Heterogeneous knowledge management technology]. Gibridnye i sinergeticheskie intellektual'nye sistemy: materialy 2-go Mezhdunarodnogo Pospelovskogo simpoziuma [Hybrid and Synergetic Intelligent Systems: Proceedings of the 2nd International Pospelovsky Symposium], pp. 45-53 (in Russ.).

Bobkov, A.V. and Yang, Xu., 2019. Methods of visual navigation in the task of moon landing. Procedia Computer Science, 150, pp. 201-207.

Deiotte, R. and La Valley, R., 2017. Comparison of Spatiotemporal Mapping Techniques for Enormous Etl and Exploitation Patterns. ISPRS Annals of Photogrammetry, Remote Sensing and Spatial Information Sciences, Volume IV-4/W2, pp. 7-13.

Dilip Kumar Choubey and Sanchita Paul, 2016. GA_MLP NN: A Hybrid Intelligent System for Diabetes Disease Diagnosis. International Journal of Intelligent Systems and Applications (IJISA), Vol.8, No.1, pp. 49-59.

Fedorenko, Y. S. and Gapanyuk, Y. E., 2018. The Neural Network with Automatic Feature Selection for Solving Problems with Categorical Variables. International Conference on Neuroinformatics. - Springer, Cham, 2, pp. 129-135.

Müller-Budack, E., Pustu-Iren, K. and Ewerth, R., 2018. Geolocation Estimation of Photos Using a Hierarchical Model and Scene Classification. Computer Vision - ECCV 2018, pp. 575-592.

Nam, N. Vo, Jacobs, N. and Hays, J., 2017. Revisiting IM2GPS in the Deep Learning Era. ICCV 2017, pp. $2640-2649$.

Suslov S. and Gavrikova K., 2017. Places of citizens' attraction in the urban space by case study of the krasnogvardeysky district of st. Petersburg. Society: politics, economics, law, pp. 17-19.

Thakare, S., Kamble, A., Thengne, V. and Kamble, U.R., 2018. Document Segmentation and Language Translation Using Tesseract-OCR. 2018 13th International Conference on Industrial and Information Systems, ICIIS 2018 - Proceedings, pp. 148-151.

Weyand, T., Kostrikov, I. and Philbin, J., 2016. Planet-photo geolocation with convolutional neural networks. European Conference on Computer Vision. - Springer, Cham, pp. 37-55.

Xinyu Zhou, Cong Yao, He Wen, Yuzhi Wang, Shuchang Zhou, Weiran He and Jiajun Liang, 2017. EAST: An Efficient and Accurate Scene Text Detector. 2017 IEEE Conference on Computer Vision and Pattern Recognitio, pp. 2642-2651.

Zaharieva, M., Zeppelzauer, M. and Breiteneder, C., 2013. Automated social event detection in large photo collections. Proceedings of the 3rd ACM conference on International conference on multimedia retrieval, pp. 167-174. 